\title{
A System of Nonlinear Differential-Difference Equations with Variable Coefficients and Its Reductions
}

\author{
Sheng Zhang ${ }^{1, a}$ and Xin Guo ${ }^{1, b}$ \\ ${ }^{1}$ School of Mathematics and Physics, Bohai University, Jinzhou 121013, PR China \\ aszhangchina@126.com, b865959684@qq.com
}

Keywords: Nonlinear differential-difference equation; Discrete spectral problem; Reduction

\begin{abstract}
Constructing integrable systems is a significant direction in soliton theory. In this paper, a new system of nonlinear differential-difference equations with variable coefficients is derived by introducing some derivable functions to the corresponding discrete spectral problems. In order to give some special cases of the derived differential-difference equations, three reductions are obtained which include Hirota's lattice equations as special cases. The processes of constructing such a system of variable-coefficient differential-difference equations and obtaining its reductions provide with a necessary help for the beginners.
\end{abstract}

\section{Introduction}

Soliton equations are a kind of special nonlinear partial differential equations, one important property of such type of equations is the existence of so-called soliton solutions (stable waves) which spread in time without changing their size or shape and interact with each other in a particle-like way [1]. There is a close relation between the existence of soliton solutions and the integrability of equations, the known research results show that all the integrable systems exist soliton solutions [2, 3]. With the development of soliton theory, constructing integrable systems becomes a significant direction in nonlinear science [4-14]. What is integrable? there is still not a unified concept. Several integrabilities have been defined, such as Lax integrability, Liouville integrability and Painlevé integrability. Generally speaking, one should indicate the system is integrable in what sense.

Since the variable-coefficient systems could describe more realistic physical phenomena than their constant-coefficient counterparts when the inhomogeneities of media and nonuniformities of coundaries are taken into account $[15,16]$, we shall construct in the present paper a system of nonlinear differential-difference equations with variable coefficients.

\section{Derivation}

Firstly, following the steps in [17] we consider the discrete spectral problems

$$
\begin{gathered}
\varphi_{1, n+1}=\left(1-T_{n} S_{n}\right)^{-1}\left(z+R_{n} S_{n}\right) \varphi_{1, n}+\left(1-T_{n} S_{n}\right)^{-1}\left(Q_{n}+\frac{1}{Z} S_{n}\right) \varphi_{2, n}, \\
\varphi_{2, n+1}=\left(1-T_{n} S_{n}\right)^{-1}\left(R_{n}+z T_{n}\right) \varphi_{1, n}+\left(1-T_{n} S_{n}\right)^{-1}\left(\frac{1}{Z}+T_{n} Q_{n}\right) \varphi_{2, n},
\end{gathered}
$$

with $\varphi_{1, n}$ and $\varphi_{2, n}$ satisfying the evolution equations

$$
\begin{aligned}
& \frac{\partial}{\partial t} \varphi_{1, n}=A_{n} \varphi_{1, n}+B_{n} \varphi_{2, n}, \\
& \frac{\partial}{\partial t} \varphi_{2, n}=C_{n} \varphi_{1, n}+D_{n} \varphi_{2, n} .
\end{aligned}
$$


Introducing shift operator $E$ defined by $E\left(\varphi_{i, n}\right)=\varphi_{i, n+1}$ and using the relation $\frac{\partial}{\partial t}\left(E \varphi_{i, n}\right)=E\left(\frac{\partial \varphi_{i, n}}{\partial t}\right)$ for $i=1,2$, we have

$$
\begin{aligned}
& \frac{\partial}{\partial t}\left(E \varphi_{1, n}\right)=\left[\left(1-T_{n} S_{n}\right)_{t}^{-1}\left(z+R_{n} S_{n}\right)+\left(1-T_{n} S_{n}\right)^{-1}\left(z+R_{n} S_{n}\right)_{t}+\left(1-T_{n} S_{n}\right)^{-1}\left(z+R_{n} S_{n}\right) A_{n}\right. \\
& \left.+\left(1-T_{n} S_{n}\right)^{-1}\left(Q_{n}+\frac{1}{Z} S_{n}\right) C_{n}\right] \varphi_{1, n}+\left[\left(1-T_{n} S_{n}\right)^{-1}\left(z+R_{n} S_{n}\right) B_{n}+\left(1-T_{n} S_{n}\right)_{t}^{-1}\left(Q_{n}+\frac{1}{Z} S_{n}\right)\right. \\
& \left.+\left(1-T_{n} S_{n}\right)^{-1}\left(Q_{n}+\frac{1}{Z} R_{n}\right)_{t}+\left(1-T_{n} S_{n}\right)^{-1}\left(Q_{n}+\frac{1}{Z} S_{n}\right) D_{n}\right] \varphi_{2, n}, \\
& E\left(\frac{\partial}{\partial t} \varphi_{1, n}\right)=\left[\left(1-T_{n} S_{n}\right)^{-1}\left(z+R_{n} S_{n}\right) A_{n+1}+\left(1-T_{n} S_{n}\right)^{-1}\left(R_{n}+z T_{n}\right) B_{n+1}\right] \varphi_{1, n} \\
& \quad+\left[\left(1-T_{n} S_{n}\right)^{-1}\left(Q_{n}+\frac{1}{Z} S_{n}\right) A_{n+1}+\left(1-T_{n} S_{n}\right)^{-1}\left(\frac{1}{Z}+Q_{n} T_{n}\right) B_{n+1}\right] \varphi_{2, n}, \\
& \frac{\partial}{\partial t}\left(E \varphi_{2, n}\right)=\left[\left(1-T_{n} S_{n}\right)_{t}^{-1}\left(R_{n}+z T_{n}\right)+\left(1-T_{n} S_{n}\right)^{-1}\left(R_{n}+z T_{n}\right)_{t}+\left(1-T_{n} S_{n}\right)^{-1}\left(R_{n}+z T_{n}\right) A_{n}\right. \\
& \left.\quad+\left(1-T_{n} S_{n}\right)^{-1}\left(\frac{1}{Z}+Q_{n} T_{n}\right) C_{n}\right] \varphi_{1, n}+\left[\left(1-T_{n} S_{n}\right)_{t}^{-1}\left(\frac{1}{Z}+Q_{n} T_{n}\right)+\left(1-T_{n} S_{n}\right)^{-1}\left(Q_{n} T_{n}\right)_{t}\right. \\
& \left.\quad+\left(1-T_{n} S_{n}\right)^{-1}\left(Q_{n}+\frac{1}{Z} R_{n}\right)_{t}+\left(1-T_{n} S_{n}\right)^{-1}\left(Q_{n}+\frac{1}{Z} S_{n}\right) D_{n}\right] \varphi_{2, n}, \\
& \quad+\left[\left(1-T_{n} S_{n}\right)^{-1}\left(Q_{n}+\frac{1}{Z} S_{n}\right) C_{n+1}+\left(1-T_{n} S_{n}\right)^{-1}\left(\frac{1}{Z}+Q_{n} T_{n}\right) D_{n+1}\right] \varphi_{2, n} . \\
& E\left(\frac{\partial}{\partial t} \varphi_{2, n}\right)=\left[\left(1-T_{n} S_{n}\right)^{-1}\left(z+R_{n} S_{n}\right) C_{n+1}+\left(1-T_{n} S_{n}\right)^{-1}\left(R_{n}+z T_{n}\right) D_{n+1}\right] \varphi_{1, n} \\
& \quad
\end{aligned}
$$

Further taking

$$
A_{n}=a_{n}^{(0)}(t)+z a_{n}^{(1)}(t), B_{n}=b_{n}^{(0)}(t)+\frac{1}{z} b_{n}^{(-1)}(t), C_{n}=c_{n}^{(0)}(t)+x c_{n}^{(1)}(t), D_{n}=d_{n}^{(0)}(t)+\frac{1}{z} d_{n}^{(-1)}(t),
$$

then using Eq. (9) to collect the coefficients of $z$ with same order in Eqs. (6-9) yields

$$
\begin{aligned}
& z^{2}: \Delta_{n} a_{n}^{(1)}(t)=0, a_{n}^{(1)}(t) T_{n}=c_{n+1}^{(1)}(t), \\
& z^{-2}: d_{n}^{(-1)}(t) S_{n}=b_{n+1}^{(-1)}(t), \Delta_{n} d_{n}^{(-1)}(t)=0, \\
& z^{1}: b_{n}^{(0)}(t)=a_{n+1}^{(1)}(t) Q_{n}, \quad b_{n}^{(0)}(t) T_{n}=c_{n+1}^{(1)}(t) Q_{n}, \\
& z^{-1}: c_{n}^{(0)}(t) S_{n}=b_{n+1}^{(-1)}(t) R_{n}, c_{n}^{(0)}(t)=d_{n+1}^{(-1)}(t) R_{n},
\end{aligned}
$$

from which we have

$$
\begin{aligned}
& a_{n}^{(1)}(t)=a(t), \quad c_{n}^{(1)}(t)=a(t) T_{n-1}, \quad b_{n}^{(-1)}(t)=d(t) S_{n-1}, \\
& d_{n}^{(-1)}(t)=d(t), \quad b_{n}^{(0)}(t)=a(t) Q_{n}, \quad c_{n}^{(0)}(t)=d(t) R_{n} .
\end{aligned}
$$

If we select

$$
d_{n+1}^{(0)}=a^{(1)}(t) T_{n} Q_{n+1}, a_{n+1}^{(0)}=d^{(-1)}(t) S_{n} R_{n+1},
$$


the following system of nonlinear differential-difference equations with variable coefficients can be obtained

$$
\begin{aligned}
& R_{n, t}=\left(1-R_{n} Q_{n}\right)\left[d(t) T_{n}-a(t) T_{n-1}\right], \\
& S_{n, t}=\left(1-S_{n} T_{n}\right)\left[a(t) Q_{n+1}-d(t) Q_{n}\right], \\
& Q_{n, t}=\left(1-R_{n} Q_{n}\right)\left[a(t) S_{n}-d(t) S_{n-1}\right], \\
& T_{n, t}=\left(1-S_{n} T_{n}\right)\left[d(t) R_{n+1}-d(t) R_{n}\right] .
\end{aligned}
$$

In particularly, when setting

$$
R_{n}=0, T_{n}=1, Q_{n}=a(t) \beta_{n}, S_{n}=1-a(t) \alpha_{n}, a(t)=d(t),
$$

Eqs. (17-20) can be reduced as a generalized Hirota's lattice equations with variable coefficients:

$$
\begin{aligned}
& \beta_{n, t}=a(t)\left(\alpha_{n-1}-\alpha_{n}\right)-\frac{a^{\prime}(t)}{a(t)} \beta_{n}, \\
& \alpha_{n, t}=a^{2}(t) \alpha_{n}\left(\beta_{n}-\beta_{n+1}\right)-\frac{a^{\prime}(t)}{a(t)} \alpha_{n} .
\end{aligned}
$$

which including the known Hirota’s lattice equations [17]:

$$
\begin{aligned}
& \beta_{n, t}=\alpha_{n-1}-\alpha_{n}, \\
& \alpha_{n, t}=\alpha_{n}\left(\beta_{n}-\beta_{n+1}\right) .
\end{aligned}
$$

as special case as long as letting $a(t)=1$.

If setting

$$
\beta_{n}=-x_{n, t}, \alpha_{n}=\mathrm{e}^{x_{n}-x_{n+1}},
$$

from Eqs. (22) and (23) we have

$$
\begin{aligned}
& \beta_{n, t}=-x_{n, t t}=a(t)\left(\mathrm{e}^{x_{n-1}-x_{n}}-\mathrm{e}^{x_{n}-x_{n+1}}\right)+\frac{a^{\prime}(t)}{a(t)} x_{n, t}, \\
& \alpha_{n, t}=\left(x_{n, t}-x_{n+1, t}\right) \mathrm{e}^{x_{n}-x_{n+1}}=a^{2}(t) \mathrm{e}^{x_{n}-x_{n+1}}\left(-x_{n, t}+x_{n+1, t}\right)-\frac{a^{\prime}(t)}{a(t)} \mathrm{e}^{x_{n}-x_{n+1}},
\end{aligned}
$$

which can be reduced as

$$
\begin{aligned}
& x_{n, t t}+a(t)\left(\mathrm{e}^{x_{n-1}-x_{n}}-\mathrm{e}^{x_{n}-x_{n+1}}\right)+\frac{a^{\prime}(t)}{a(t)} x_{n, t}=0, \\
& x_{n, t}-x_{n+1, t}+\frac{a^{\prime}(t)}{a^{3}(t)+a(t)}=0 .
\end{aligned}
$$

Integrating Eq. (25) with respect to $t$ one, we have

$$
x_{n}-x_{n+1}=\log \frac{\left[1+a^{2}(t)\right]^{\frac{1}{2}}}{a(t)},
$$

then substituting Eq. (26) into Eq. (24) yields

$$
x_{n, t t}+\frac{a^{\prime}(t)}{a(t)} x_{n, t}=0 .
$$


Solving Eq. (27), we obtain a special solution of Eqs. (24) and (25):

$$
x_{n}=c_{0}+c_{1} \int \frac{1}{a(t)} \mathrm{d} t .
$$

\section{Conclusion}

By introducing some derivable functions to the corresponding discrete spectral problems, a system of nonlinear differential-difference equations with variable coefficients is derive. To the best of our knowledge, this system of differential-difference equations has not been reported in literature. Besides, three reductions of the derived system of differential-difference equations are obtained which include Hirota's lattice equations as special cases. How to employ the method used in this paper to construct some other nonlinear differential-difference equations with variable coefficients is worthy of study. This is our tasks in the future.

\section{Acknowledgements}

This work was supported by the Natural Science Foundation of Educational Committee of Liaoning Province (L2012404) of China, the PhD Start-up Funds of Bohai University (bsqd2013025) and Liaoning Province of China (20141137), the Liaoning BaiQianWan Talents Program (2013921055) and the Natural Science Foundation of China (11371071).

\section{References}

[1] G. Teschl: Jahresber. Deutsch. Math.-Verein. Vol. 103 (2001), p. 149

[2] S.Y. Lou and X.Y. Tang: Method of Nonlinear Mathematical Physics (Science Press, Beijing 2006)

[3] S. Zhang, Y.Y. Zhou, IAENG Int. J. Appl. Math. Vol. 44 (2014), p. 177

[4] M.J. Ablowitz and P.A. Clarkson: Soliton, Nonlinear Evolution Equations and Inverse Scattering, (Cambridge University Press, Cambridge 1991)

[5] G.Z. Tu, J. Math. Phys. Vol. 30 (1989), p. 330

[6] Y.F. Zhang, Phys. Lett. A Vol. 317 (2003), p. 280

[7] F.C. You and T.C. Xia, Chaos Soliton. Fract. Vol. 36 (2008), p. 953

[8] S. Zhang, B. Xu and H.Q. Zhang, Int. J. Comput. Math. Vol. 91 (2014), p. 1601

[9] F.J. Yu, Abstr. Appl. Anal. Vol. 2014 (2014), 146537

[10]S. Zhang and D. Wang: Therm. Sci. Vol. 18 (2014), p. 1555

[11]Y.F. Zhang and B.L. Feng, Commun. Nonlinear Sci. Numer. Simulat. Vol. 16 (2011), p. 3045

[12]F.J. Yu, Phys. Lett. A Vol. 375 (2011), p. 1504

[13]Y.F. Zhang, Commun. Theor. Phys. Vol. 56 (2011), p. 805

[14]F.J. Yu and L. Li, Phys. Lett. A Vol. 372 (2008), p. 3548

[15]Y. Liu, Y.T. Gao, Z.Y. Sun, and X. Yu: Nonlinear Dyn. Vol. 66 (2011), p. 575

[16]S. Zhang and B. Cai: Nonlinear Dyn. Vol. 78 (2014), p. 1593

[17]Y.S. Li: Soliton and Integrable System (Shanghai Scientific and Technological Education Publishing House, Shanghai 1999) 